\title{
PPPM in cardiovascular diseases
}

\section{PPPM IN CARDIOVASCULAR PATHOLOGIES: JAPANESE HEALTH SUCCESS IN COMBATING CARDIOVASCULAR DISEASES}

Iso $H$

Osaka University, Japan

Japan has achieved the top-rank for longevity in the world in 2010, the average life expectancy is 79.6 years for men and 86.4 years for women. The Japanese longevity is primarily due to the control of acute infectious diseases, malnutrition and tuberculosis between the 1940s and 1960 s, and the control of cardiovascular disease since the 1960s.

This health success story in Japan is unique in terms of the reduction of cardiovascular disease, both of stroke and ischemic heart disease (IHD) between the 1960s and 2000s. That mortality decline was attributable to reductions of blood pressure and smoking, in spite of increases in dyslipidemia and diabetes mellitus.

Other developed countries such as European countries and the United States still have an IHD epidemic with the high prevalence of obesity, diabetes mellitus, and dyslipidemia (high triglycerides and low HDL-cholesterol), while the burden of IHD is smaller but is increasing with population aging in Japan. On the other hand, many developing countries, while in the process of conquering malnutrition and infectious disease, have encountered emerging cardiovascular disease along with rapidly increasing prevalence of obesity, diabetes, hypertension, and dyslipidemia.

The remarkable difference in cardiovascular profiles between Japan and the North American and European countries may be attributable to different proportions of two major vascular pathologies occurring in small and large arteries. Arteriosclerosis (small vessel pathology) has been dominant in Japanese populations, reflecting high stroke mortality, while the same is true for atherosclerosis (large vessel pathology), reflecting high IHD mortality in European and US populations. The recent rise in IHD incidence among urban middle-aged men, however, suggests a potential increase in atherosclerosis among Japanese subpopulations.
From the viewpoint of predictive, preventive, and personalized medicine, the Japanese health success story in the prevention of cardiovascular disease may be of value for many developed and developing countries in building effective population and individual strategies for the prevention and control of cardiovascular disease. To this end, we reviewed mortality and morbidity trends in cardiovascular disease, risk factors, and related Japanese lifestyles over the past decades, and the public health and individual personalized approaches for prevention and control of cardiovascular disease.

The combination of public health and personalized treatment activities to enhance sodium reduction, balanced diets, smoking prevention/cessation as well as hypertension detection and treatment have contributed to the reduction of cardiovascular disease, demonstrated by a communitybased stroke prevention program.

The health success story, however, contains an underlying concern about future health threats, the plateaued IHD mortality decline and increased IHD incidence among urban middle-aged men, probably due to increased dyslipidemia and diabetes. The IHD incidence and mortality trends need to be monitored because of a potential future problem for predictive, preventive, and personalized medicine.

\section{THE INFLUENCE OF SULFORAPHANE ON VASCULAR HEALTH AND ITS RELEVANCE TO NUTRITIONAL APPROACHES TO PREVENT CARDIOVASCULAR DISEASE}

Evans $P C$

British Heart Foundation Cardiovascular Sciences Unit, National Heart and Lung Institute, Imperial College London, UK

Atherosclerosis, a lipid-driven inflammatory disease of arteries, is the leading cause of death in the Western world. It is promoted by oxidation of low-density lipoproteins which enhances vascular inflammation and the accumulation of lipid-laden macrophages (foam cells). The corollary is that diets that stimulate endogenous anti-oxidants may protect against atherosclerosis. 
My group has recently examined whether sulforaphane, a compound generated by the consumption of green vegetables such as broccoli, can protect arteries from inflammation and injury. Our work using cultured endothelial cells and murine models revealed that sulforaphane can suppress inflammatory activation of arterial EC. The mechanism relies on activation of the transcription factor $\mathrm{Nrf2}$ which induces numerous antioxidant enzymes. Thus our studies reveal a novel molecular mechanism that may contribute to the beneficial effects of green vegetable intake on cardiovascular health. A deeper understanding of vascular responses to sulforaphane may inform nutritional approaches to prevent vascular inflammation and atherosclerosis.

\section{CARDIO-METABOLIC RISK PREDICTION SHOULD BE SUPERIOR TO CARDIOVASCULAR RISK ASSESSMENT IN PRIMARY PREVENTION OF CARDIOVASCULAR DISEASES}

Rosolova H, Nussbaumerova B

Charles University in Prague, Medical Faculty in Pilsen, Czech Republic

Atherosclerosis is considered the largest non-infectious epidemic of the last 50 years worldwide. Cardiovascular atherosclerotic diseases represent the main cause of death in the majority of developed and also developing populations. Although major progress has been made in the management of the classical modifiable cardiovascular risk factors (e.g., hypertension, hypercholesterolemia), sedentary lifestyle and intake of high energy diet conduce to an increasing prevalence of overweight, obesity, metabolic disorders, and type 2 diabetes mellitus. Subjects with these metabolic disorders have a significantly increased risk for premature atherosclerosis and, conversely, patients with established cardiovascular diseases have a high risk for metabolic disorders associated with insulin resistance. That is why cardio-metabolic risk prediction should be superior in the primary prevention of atherosclerosis and cardiovascular diseases.

Up-to-date strategies in the primary prevention of cardiovascular diseases according to the European Guidelines on cardiovascular disease prevention, especially the high-risk strategy approach, are being implemented.
Individual cardiovascular and better cardio-metabolic risk assessment represents the basic approach in the individualized primary prevention of cardiovascular diseases and type 2 diabetes mellitus. Metabolic abnormalities such as abdominal fatty tissue distribution, atherogenic dyslipidemia defined as elevated triglycerides concentration and reduced high-density lipoprotein cholesterol, impaired glucose homeostasis and elevated blood pressure cluster in subjects with insulin resistance; these clustered risk factors were termed as "metabolic syndrome" (MS). The presence of MS usually does not impact the risk in subjects at high risk, whereas the presence of MS in subjects at low or intermediate risk significantly increases the probability of developing CVD, making it imperative to improve their global cardio-metabolic risk assessment.

Various biomarkers have been investigated to improve the prediction of individual cardio-metabolic risk over the last two decades. Especially high sensitivity-C reactive protein, albuminuria and N-terminal pro-brain natriuretic peptide, seems to be useful in primary prevention. There are many individual combinations of known risk factors and emerging risk factors and our ultimate goal is to identify some markers or group of markers to more precisely determining absolute personalized risk for atherosclerosis and/or metabolic disorders and then institute personalized preventive measures early enough to stop the development of the disease

Imaging procedures such as computed tomography angiography, coronary arteries calcium score assessment, magnetic resonance imaging, etc. improve the prediction of cardiometabolic risk. However, most of them are not suitable for primary preventive screening in the general population, ultrasound carotid intima-media thickness measurement is a non-invasive, reproducible, inexpensive, screening test for assessment of a subclinical atherosclerosis.

The many barriers to better cardiovascular disease and type 2 diabetes prevention are still evident. In fact, they reflect the difficulty in making accurate cardiometabolic risk estimation in the clinical setting, particularly in the intermediate-risk subjects. Clinically applicable genetic markers will improve personalized approach in diseases prediction and prevention.

Figure: Predicting cardiometabolic risk in the $21^{\text {st }}$ century: 


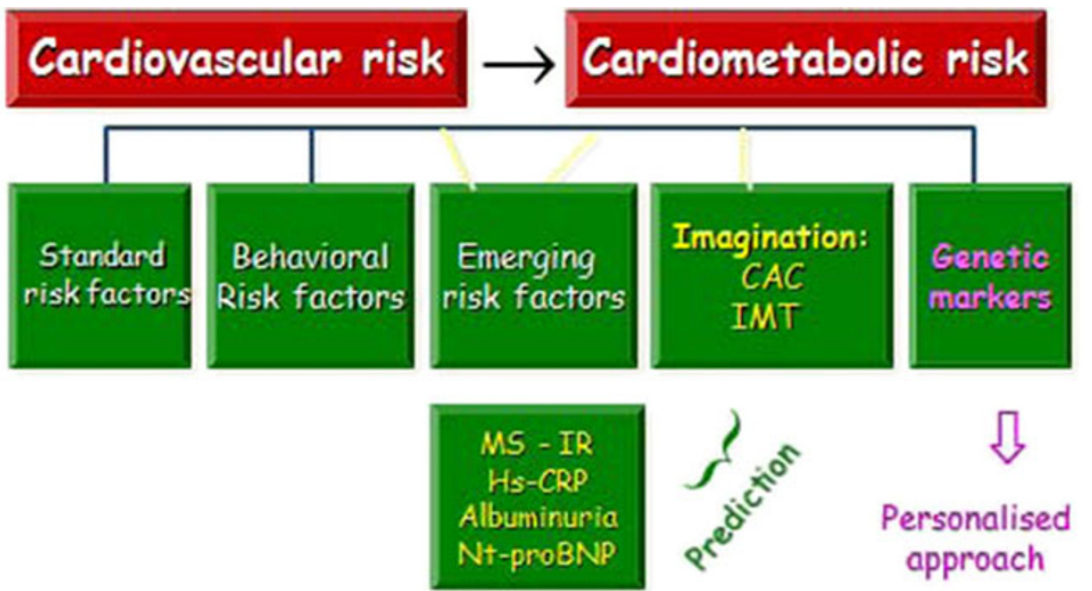

\begin{abstract}
Abbreviations:
CAC coronary arteries calcium, IMT intima media thickness, MS-IR metabolic syndrome - insulin resistence, Nt-proBNPN- terminal probrain natriuretic peptide.
\end{abstract}

\section{DEGENERATIVE VALVE DISEASE: RISK ASSESSMENT, PERSONALISED TREATMENTS, PROGNOSIS}

Yeghiazaryan $K^{1}$, Skowasch $D^{2}$, Bauriedel $G^{3}, H$ Schild $H^{1}$, Golubnitschaja ${ }^{1}$

${ }^{1}$ Department of Radiology, Rheinische Friedrich-WilhelmsUniversity of Bonn, Germany

${ }^{2}$ Department of Internal Medicine II - Cardiology, Rheinische Friedrich-Wilhelms-University of Bonn, Germany

${ }^{3}$ Department of Internal Medicine III, Hospital Schmalkalden, Germany

\section{Clinical assessment of aortic stenosis}

Aortic stenosis (AS) is the most frequent valvular heart disease. Its prevalence increases with age, and has been reported between 2 to $4 \%$ in a population $\geq 65$ years old. Severe AS results in concentric left ventricular hypertrophy, and ultimately, the heart dilates and fails. During a long period of time, patients remain asymptomatic, acute complications are rare. Disease progression may be well monitored by Doppler-echocardiography, an accurate noninvasive measurement of the stenosis severity. After the onset of symptoms the outcome becomes poor with a survival rate not longer than 2-3 years, when valve replacement becomes a strongly recommended emergency approach.

Molecular signature of valvular degeneration

A number of gene have been detected as highly affected by altered expression rates in degenerating valvular tissue: these are potassium voltage-gated channel-1, cardiotrophin, cardiac myosins, metalloproteinases, endothelins, neuropilins, caveolins, progesterone-, vasopressin-, tumor-necrosisfactor- and adrenergic-receptors. Moreover, whereas wellexpressed hepatic lipase has been demonstrated in calcified valves, no traces of its expression could be detected in noncalcified tissue. Those gene products should be taken into account for a predictive diagnosis and considering individualised therapeutic approaches.

A functional link between cell cycle control and calcification of aortic valves: potential diagnostic and prognostic targets

The coordinated suppression of checkpoint genes in calcified aortic valves at both transcription (A) and translation (B) levels is represented in Fig. 1. Both cellularity and number of macrophages are significantly increased in calcified tissue (see Fig. 1C and Fig. 1D, respectively).

Pronounced up-regulation of both genes in non-calcified in contrast to their down-regulation in calcified degenerated valvular tissue indicates the central regulatory role of checkpoint genes in keeping functional the valvular cells. 
The coordinated activation of both $\mathrm{G}_{1}$ and $\mathrm{G}_{2}$ dependent checkpoint genes may be an attribute of the valvular tissue resistance against the calcification processes. These data should be taken into consideration to design novel therapeutic approaches targeted at pro-calcification mechanisms in the heart.
A

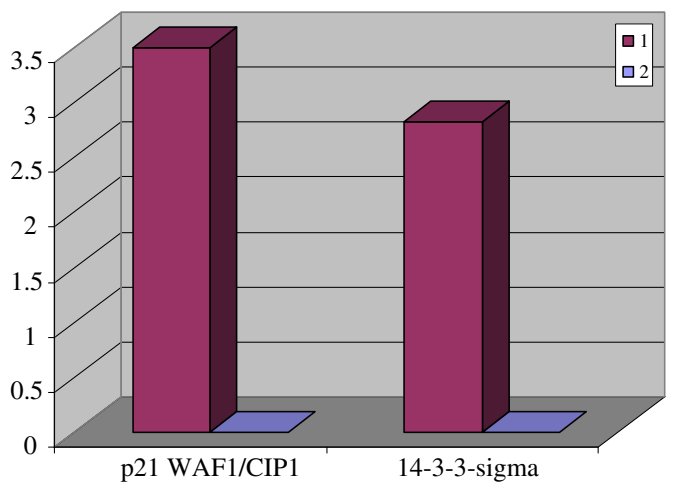

\section{B}

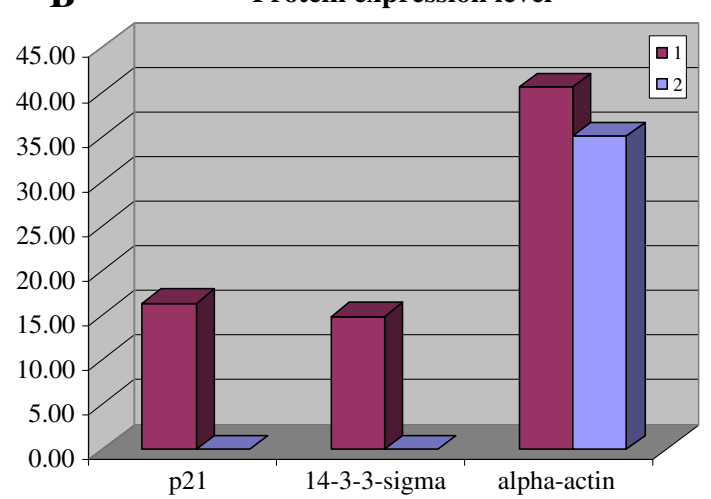

C

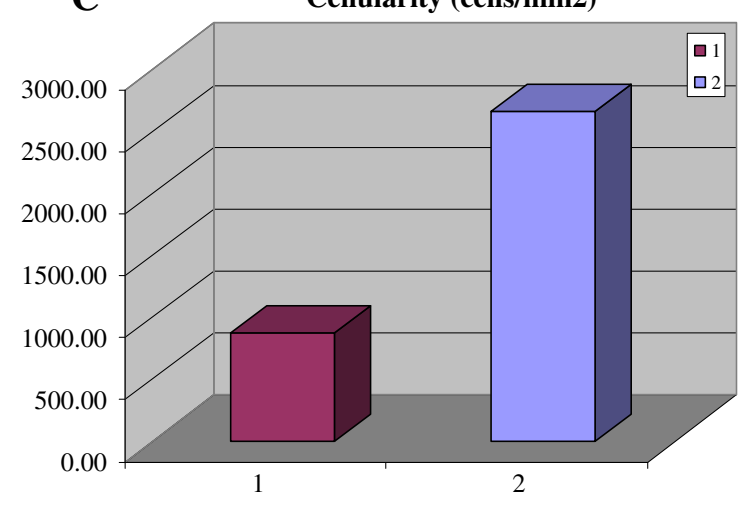

D

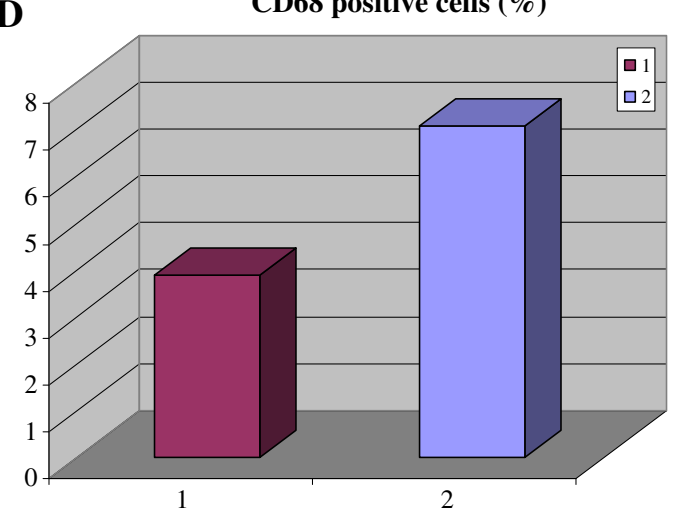

Fig. 1 Comparative analysis in two groups of patients with non-calcified (1) versus calcified (2) degenerative aortic valves. (A) Comparative gene expression analysis (mRNA level in relative units) of $\mathrm{p} 21^{\mathrm{WAF} 1 / \mathrm{CIP} 1}$ and 14-3-3-sigma. Quantitative Real-Time-PCR was applied. (B) Comparative analysis of protein expression levels (in relative units) of P21 ${ }^{\mathrm{WAF} 1 / \mathrm{CIP} 1}, 14-3-3$-sigma and alpha-actin. (C) Comparative analysis of cellular density (in relative units). (D) Comparative analysis of macrophages (in relative units)

\section{Risk assessment: factors involved in degenerative valve disease}

Traditional cardiovascular risk factors such as lipid disorders, diabetes, arterial hypertension, smoking and male gender increase the incidence of AS: more frequently the combination of them is usually observed in this cohort of patients. No longer considered as a natural consequence of ageing, DAVD is the result of actively driven pathological processes including programmed (dys)regulation of target genes, metabolic alterations, inflammatory cell infiltration, subcellular disruption, and consequent tissue degeneration, calcification and remodelling. Molecular / cellular processes attributed to DAVD are usually triggered "down-stream" towards a metabolic impairment (diabetes mellitus, hypercholesterolemia, hypercalcaemia, leanness), hormonal deregulation (hyperparathyroidism), hypertension, and extreme stress conditions such as tobacco use and environmental stress factors. Thus, an inverse relation- 
ship was demonstrated between body mass index and DAVD incidence: calcific changes were more frequently observed in lean people even independently of the risk factor of age, and, therefore, cannot be explained by leanness frequently observed in patients with highly advanced age. These facts indicate, further, an association of DAVD with metabolic disorders causing weight loss such as osteoporosis. Similarly to diabetic patients, smokers also suffer from highly increased ROS production leading to enhanced incidence of DAVD, although specific pathomechanisms deserve further clarification. Dysregulation of angiotensin-II metabolism and activity of angiotensinspecific receptors is considered to be the key molecule in the pathomechanisms that underlie DAVD in hypertension.

\section{Individualised treatment of aortic stenosis and prognosis}

For the aortic stenosis, a variable disease progression is characteristic proposing successful treatments by individualised therapeutic approaches to prevent or at least slow down the disease progression For examples, the beneficial effects by statin appear due to its pleiotropic and/or anti-inflammatory properties but independently from lowering cholesterol impacts. Further, lowering LDL-cholesterol levels do not halt the progression of aortic stenosis in patients with mild to moderate aortic-valve disease. The fact that angiotensin converting enzyme (ACE) and angiotensin II can be found in sclerotic but not in normal aortic valves indicates an important role of the renin-angiotensin system in the pathogenesis of AS. Consequently, ACE inhibitors slow down the calcium accumulation in aortic valves; they are considered for their therapeutic application to effectively prevent both hypertension and DAVD. Independently from individual risk factors, the crucial role of metalloproteinases in the central pathomechanisms of the progressive tissue remodelling during the chronic development of DAVD is well recognised. Novel therapeutic interventions consider, therefore, metalloproteinases as the preferred target to delay or even prevent the progression of DAVD.

\section{Outlook}

Molecular attributes characteristic for early stages of valvular degeneration represent reliable predictive biomarkers and targets for more effective individualised treatment approaches before the pathology is clinically manifested. Risk factors should be considered individually. The characteristic molecular signature is one of them. On average, patients without diagnosed risk factors undergo a re-operation 9-10 years after aortic valve replacement (AVR). This period is halved for patients demonstrating at least two of following risk factors: smoking, Diabetes mellitus, risk by gender (females), high cholesterol levels. Therefore, individualised targeted measures would be highly effective in prevention of AVD and reoperation after AVR. Pathologyand stage-specific molecular patterns should be taken into consideration for the reliable prediction, individualised treatment algorithms and correct prognosis.

\section{References:}

1. Yeghiazaryan K, Skowasch D, Bauriedel G, Schild HH, Golubnitschaja O. Degenerative valve disease and bioprostheses: risk assess-ment, predictive diagnosis, personalised treatments. EPMA J. 2011;2:91-105.

\section{STEM CELLS THERAPY FOR CARDIOVASCULAR REPAIR IN ISCHEMIC HEART DISEASE: HOW TO PREDICT AND SECURE OPTIMAL OUTCOME?}

\section{Kastrup J}

The Heart Centre, Rigshospitalet University Hospital Copenhagen, Denmark

Coronary artery disease is a growing problem worldwide. Early treatment with stabilizing drugs and revascularization by percutaneous coronary intervention or by-pass surgery has reduced the mortality significantly, but it is still the most common cause of death and a major cause of hospital admissions in industrialised countries.

Regenerative medicine with vascular growth factor and stem cell therapy within the last decennium had awoken great interest and has been tested in clinical trials in patients with ischemic heart disease. The aim is to induce growth of new blood vessels or replacement of damaged myocardial cells either directly by trans-differentiation of stem cells or by a paracrine effect of cytokines secreted from the stem cells.

Within cardiology most focus has been on bone marrow derived haematopoietic stem cells, which have the potential to differentiate into endothelial cells and create new blood vessels and hereby be involved in myocardial vasculogenesis. Several clinical safety and efficacy studies with mononuclear cell solutions and selected endothelial progenitor cells from the bone marrow have been conducted in patients with acute myocardial infarction, chronic ischemic 
heart disease or ischemic heart failure. However, the results have been conflicting. Some but not all studies suggested a beneficial effect on myocardial function and symptoms.

These conflicting results have changed focus towards the use of more specific stem cell lines. Some groups have focused on the multi-potent mesenchymal stromal cell from the bone marrow or adipose derived stem cell, which easily can be isolated from the bone marrow and abdominal adipose tissue, in vitro culture expanded, and stimulated to differentiate into different cell lines as endothelial, cardiac or other cell types.

This contribution is based on results from conducted clinical stem cell trials in patients with ischemic heart disease for elucidating how to predict and personalize this new treatment approach.

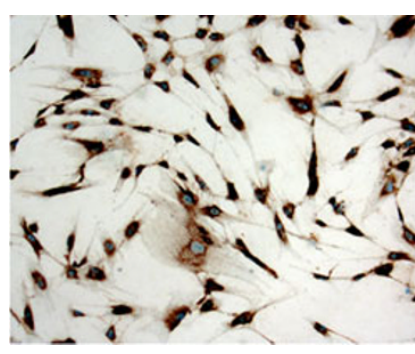

Week 1

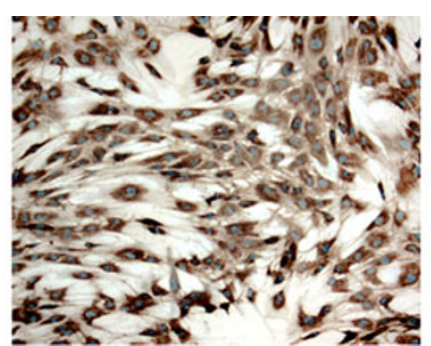

Week 2

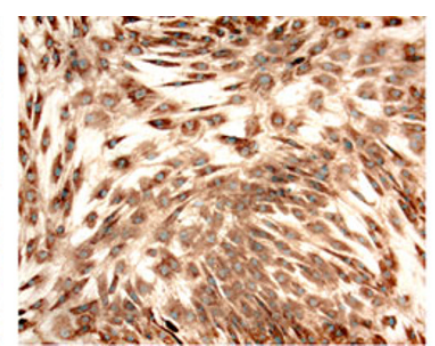

Week 3

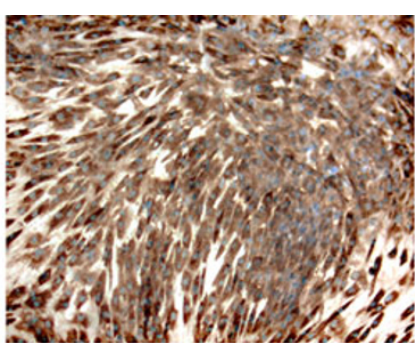

Week 5

Fig. Collagen staining: cultivation of mesenchymal stromal cells from the bone marrow for clinical therapy. The cells needs several weeks of proliferation to reach a sufficient number for clinical treatment. The cells are step-by-step becoming more confluent during cultivation. They are harvested for treatment or for the next expansion passage, when they are approximately 80-90\% confluent (week 5)

\section{RELATIONSHIP BETWEEN MAINTENANCE WARFARIN DOSE AND THE NUMBER DRUGS USED IN CONJUNCTION WITH IN PATIENTS AFTER HEART VALVE REPLACEMENT: VALUE OF CYP2C9 GENOTYPE}

Sychev DA, Arslanbekova SM, Kazakov, RE, Dmitriev VA, Polikarpova OA, Golukhova EZ

Department of Clinical Pharmacology, Sechenov's First Moscow State Medical University, Moscow, Russia

Department of noninvasive arrhythmology, Bakulev's Scientific Center of Cardiovascular Surgery RAMS, Moscow, Russia

Actuality: The magnitude of the maintenance warfarin dose influenced by various factors, including genetic (genetic polymorphisms CYP2C9, VKORC1). In patients after heart valve replacement dose warfarin is selected on the background of a large number of drugs (antibacterial, antifungal drugs, analgetics). It may also affect the value of a maintenance warfarin dose in these patients.

Materials and methods: The study included 34 patients after heart valve replacement at the age of $47 \pm 14$ years, 21 men and 13 women. Warfarin dose was adjusted against the backdrop of $11,5 \pm 2,2$ drugs. All patients conducted by CYP2C9 genotyping by PCR-RFLP after preliminary DNA extraction from leukocytes. Correlation analysis was performed by Pierce method.

Results: When analyzing the entire group of patients revealed unreliable inverse correlation between the magnitude of the maintenance warfarin dose and the amount of co-administered drug: $\mathrm{r}=-0,25, \mathrm{p}=0,1628$. Among the 34 patients 22 were carrying the genotype CYP2C9*1/*1, 11 patients-CYP2C $9 * 1 / * 2,1$ patient-CYP2C $9 * 1 / * 3$. The analysis of a subgroup of patients with genotype CYP2C $9 * 1 / * 1$, revealed significant inverse correlation between the magnitude of the maintenance warfarin dose and the amount of the co-administered drug: $r=-0,53, p=$ 0,01 . This correlation in a subgroup of patients with genotypes non-CYP2C9*1/*1 (genotypes CYP2C $9 * 1 / * 2$ and $\mathrm{CYP} 2 \mathrm{C} 9 * 1 / * 3$ ) was non-significant: $\mathrm{r}=-0.15, \mathrm{p}=0.68$. Conclusions: Significant correlation between the magnitude of the maintenance warfarin dose and the amount of the administered drug was detected only in patients after heart valve replacement with the genotype CYP $2 \mathrm{C} 9 * 1 /{ }^{*} 1$. This suggests that these patients the magnitude of a warfarin maintenance dose of is primarily dependent on drugs interaction, apparently due to inhibition of CYP2C9. At patients with genotypes non-CYP2C $9 * 1 / * 1$ (genotypes CYP2C $9 * 1 / * 2$ and CYP2C $9 * 1 / * 3$ ) such a relationship was not found. This may be due to the fact that in the genetically determined low activity of CYP2C9, the influence of the drug inhibitors is rather non-significant.

\author{
ASSOCIATIONS OF IMPAIRED GLUCOSE \\ METABOLISM AND DYSLIPIDEMIA \\ WITH CARDIOVASCULAR DISEASE: WHAT HAVE \\ WE LEARNED FROM JAPANESE COHORT \\ STUDIES FOR INDIVIDUALIZED PREVENTION \\ AND TREATMENT?
}




\section{Kokubo Y}

Department of Preventive Cardiology, National Cerebral and Cardiovascular Center, Osaka, Japan

Metabolic disorder is a modifiable risk factor for cardiovascular disease, and lifestyle modification is the key to improving metabolic disorder. This session reviews the associations of impaired glucose metabolism and dyslipidemia with cardiovascular disease in Japanese cohort studies.

Diabetes mellitus has become a major public health problem and been shown to be a risk factor for coronary heart disease and ischemic stroke in both Western and Japanese populations. The incidence of hyperglycemia has increased for the past three decades in Japan. Impaired fasting glucose or borderline diabetes is a risk factor for coronary heart disease especially in women, and impaired glucose tolerance is a risk factor for cardiovascular disease mortality. However, impaired glucose tolerance has not been observed as a risk factor for the incidence of ischemic stroke or coronary heart disease in Japan. An association between impaired fasting glucose and pre-hypertension found in an urban Japanese population (approximately $10 \%$ of the adult urban population) emphasized the combined risk of these factors for incidence of cardiovascular disease.

Mean levels of total cholesterol have been increasing during the last three decades in Japan. The associations with all-cause mortality were observed for both the lower and higher levels of cholesterol. The Japanese evidence for the positive association of total cholesterol with coronary heart disease is similar to that in the West. High-density lipoprotein (HDL) cholesterol levels were inversely related with ischemic stroke. Positive associations between serum triglyceride levels and coronary heart disease and ischemic stroke have also been observed in Japanese populations. Higher levels of low-density lipoprotein (LDL) cholesterol have been shown to increase the risk of coronary heart disease and atherothrombotic infarction, whereas lower levels of LDL cholesterol may increase the risk of intracerebral hemorrhage in Japan, as elsewhere. However, only one cohort study shows the positive association between LDL cholesterol levels and risk of atherothrombotic infarction. The association between non-HDL cholesterol and cardiovascular disease is similar to that for LDL cholesterol. Recently, the lectin-like oxidized LDL receptor 1 (LOX-1) has been implicated in atherothrombotic diseases. Higher LOX-index levels, obtained by multiplying the circulating concentration of LOX-1 ligands containing apolipoprotein B by that of the soluble form of LOX-1, were associated with an increased risk of cerebral infarction and coronary heart disease.

In conclusion, the Japanese diet has been rapidly changing in recent decades, as reflected in many health indicators such as cholesterol levels, and both impaired glucose metabolism and dyslipidemia are emerging as important risk factors for cardiovascular disease in the Japanese population. In order to reduce the risk of cardiovascular disease, persons with metabolic disorder should reduce not only the factors contributing to the metabolic disorder but also other cardiovascular risk factors, and improve their lifestyle, aiming at cessation of smoking, moderation of drinking, and weight reduction if appropriate.

\section{ADIPOSITYAND CARDIOVASCULAR DISEASE RISK IN JAPAN: -IMPLICATIONS TO PUBLIC HEALTH SCREENING PROGRAM OF OBESITY FOR THE PREVENTION OF CARDIOVASCULAR DISEASE \\ Yatsuya $H,{ }^{1}$ Yamagishi $K,{ }^{2}$ Iso $H^{3}$ \\ ${ }^{1}$ Department of Public Health, Nagoya University Graduate School of Medicine, Japan \\ ${ }^{2}$ Department of Public Health Medicine, Graduate School of Comprehensive Human Sciences, and Institute of Community Medicine, University of Tsukuba, Tsukuba, Japan \\ ${ }^{3}$ Public Health, Department of Social and Environmental Medicine, Osaka University Graduate School of Medicine, Osaka, Japan}

Obesity is a major health concern for most of the world given its already high prevalence. Obesity prevalence has doubled or tripled in many countries since 1980, and is still projected to increase. Even in a relatively lean population like the Japanese, an association has been established between adiposity and cardiovascular disease (CVD) risk factors, including hypertension, diabetes mellitus, and dyslipidemia. However, there is a sizeable proportion of individuals who are at an increased CVD risk state without being currently overweight/obese. Public health activities to prevent CVD and promote population health should not overlook this fact.

Although a current public health screening and intervention program in Japan that started in 2008 has considerably raised public awareness of obesity and metabolic syndrome, it overemphasized measures to deal with abdominal obesity to the detriment of predictive diagnosis and targeted prevention. Consequently, the intervention program has tended to overlook the opportunity to intervene with nonobese high risk individuals, such as lean hypertensives. More extensive communication is necessary among medical and public health professionals as well as health and insurance administrators to amend the act for this program. We conclude that further implementation and promotion of activities to bring about meaningful changes in the obesity 
trend in rural and urban communities are needed. However, such activities should be harmonized with other domains of CVD prevention activities.

\section{N-TERMINAL FRAGMENT OF THE PROHORMONE BRAIN-TYPE NATRIURETIC PEPTIDE (NT-PROBNP) AND CARDIOVASCULAR RISK FACTORS AMONGST JAPANESE POPULATION: THE CIRCULATORY RISK IN COMMUNITIES STUDY}

Yamagishi $K^{1,2}$, Kitamura $A^{2}$, Kiyama $M^{2}$, Okada $T^{2}$, Ohira $T^{2,3}$, Imano $H^{2,3}$, Cui $R^{2,3}$, Noda $H^{2,3}$, Umesawa $M^{1,2,4}$, Shimizu $Y^{2}$, Ishikawa $Y^{2}$, Iso $H^{2,3}$

${ }^{1}$ University of Tsukuba, Tsukuba, Japan

${ }^{2}$ Osaka Medical Center for Health Science and Promotion, Osaka, Japan

${ }^{3}$ Osaka University, Suita, Japan

${ }^{4}$ Ibaraki Prefectural University of Health Sciences, Ami, Japan

Background: N-terminal fragment of the prohormone braintype natriuretic peptide (NT-proBNP) is known as a biomarker of heart failure, but its distribution and correlates were not elucidated amongst Japanese general population. Methods: The subjects were 1,579 men and women ages 30 or more in a Japanese community from the Circulatory Risk in Communities (CIRCS) 2010 survey. We measured several cardiovascular risk factors in the survey as well as serum NT-proBNP levels, and compared age and sexadjusted levels or prevalence of these factors according to NT-proBNP $(\mathrm{pg} / \mathrm{ml})$ categories $(0-54,55-124,125-499$, 500-999 and 1000 or more).

Results: NT-proBNP distributed $57 \%$ for $0-54,28 \%$ for $55-$ $124,13 \%$ for $125-499,2 \%$ for $500-999$, and $1 \%$ for 1000 or more. NT-proBNP was correlated with age across the NTproBNP categories below 500, and reached its plateau thereafter. NT-proBNP was also correlated with the prevalence of hypertension below NT-proBNP of 1000, and dropped down thereafter. A prevalence of diabetes and heart diseases, histories of arrhythmias and heart failure, prevalence and history of atrial fibrillation, and symptoms of nocturnal dyspnoea and dyspnoea on exertion were also correlated generally with NT-proBNP. Abnormal electrocardiogram (defined as abnormal Q, ST-T change, complete left bundle branch block and/or atrial fibrillation) was strongly correlated with NT-proBNP: age and sex-adjusted prevalence were $2 \%$ for $0-54,4 \%$ for $55-124,16 \%$ for $125-499,76 \%$ for $500-999$ and $88 \%$ for 1000 or more. Body mass index, swollen legs at the end of the day, coughing, phlegm and/or wheezing have not correlated with NT-proBNP.

Conclusion: NT-proBNP has correlated with age, prevalence of hypertension, diabetes and heart diseases, histories of arrhythmias and heart failure, prevalence and history of atrial fibrillation, symptoms of nocturnal dyspnoea and dyspnoea on exertion, and especially with abnormal electrocardiogram amongst Japanese population.

\section{OPTIMISATION OF STROKE CARE IN CONCEPT OF PREDICTIVE, PREVENTIVE AND PERSONALISED MEDICINE}

Polivka $\mathrm{J}^{1}$, Topolcan $\mathrm{O}^{2}$, Polivka $\mathrm{J} \mathrm{jr}^{3}$

Faculty of Medicine of Charles University in Pilsen and

${ }^{1}$ Teaching Hospital Plzen, Dpt. of Neurology,

${ }^{2}$ Immuno-Analytic Laboratory,

${ }^{3}$ West-Bohemian University Plzen, Czech Republic

The progress of stroke care is dated from 1995 when thrombolytic therapy was implemented. Stroke is nonhomogenous group of patophysiological entities including atherothrombosis, hemorrhage, embolism and other less common conditions. Stroke is medical emergency. Its successful treatment relies on clinical diagnosis, neuroimaging techniques and early treatment. Secondary as well as primary prevention are near identical to cardiovascular diseases. Great effort in acute stroke management is focused on correct and rapid diagnosis and maximal shortening of "onset to needle time". In secondary and primary prevention there is nowadays the effort to pass from the wide general arrangements to the effective individually-aimed approach.

A quantity of stroke biomarkers is investigated. Lp-PLA2 (Lipoprotein-associated phospholipase A2) or ADMA (Asymmetric dimethylarginine) are associated with the increase in stroke occurrence. MMP9 (Matrix metalloproteinase 9) could be used for the acute stroke diagnosis, its concentrations have also been related to infarct size, poor neurological outcome and hemorrhagic complications. S100Beta belongs to the family of calcium-mediated proteins (S100 proteins) and is highly specific to nervous tissue. It could be used as a marker of blood-brain barrier dysfunction. NMDA (N-methyl-D-aspartic acid) receptors are heterogeneous on neuronal cells throughout the brain. The production of NMDA receptor antibodies (NR2Abs) is linked to the immune system reaction after the ischemic event. GFAP is a protein of monomeric filament specific to the astrocytes and could be hypothetically used for the differentiation between hemorrhagic and ischemic stroke. The PARK7 protein plasma concentrations demonstrated significant increase just after stroke onset. Many other biomarkers are used for stroke diagnosis and risk prediction research, such as hs-CRP, VCAM (Vascular cell adhesion molecule), MCP-1 (Monocyte chemoattractant protein-1), SAA (Serum amyloid A), IL-6 (Interleukin-6) as the markers of inflammation, dyslipidemia and endothelial damage like apolipoproteins ApoC1, ApoC3, BNP (Brain 
natriuretic peptide), Growth factors like BDNF (Brainderived neurotrophic factor), Endothelial damage like MBP (Myelin basic protein), NSE (Neurone specific enolase).

Blood biomarker models with multiple agent approach for the diagnoses of stroke have been already investigated such as the model with BNP/CRP/D-Dimer/MMP-9/S-100B, $\mathrm{S} 100 \mathrm{~B} / \mathrm{VCAM} / \mathrm{vWF}$ (von Willebrand factor or S100B/ NSE/GFAP/APC-PCI, Activated protein $\mathrm{C}$ - protein $\mathrm{C}$ inhibitor complex) as a method of differentiating ischemic stroke and intracerebral hemorrhage.

Genetic and genomic factors which are related to vascular disease also reflect the predisposition to stroke. Gene expression profiling and mRNA profiling of the blood may be important for the prediction of stroke risk. The risk variants of gene expression related to stroke are the 20210A variant of the prothrombin gene or the $1691 \mathrm{~A}$ variant of the factor 5 Leiden gene.

The possibilities of using the conventional and the "new" biomarkers in acute diagnostics, prediction of the severity of cerebral lesion, of the complications of acute stroke, and in the optimisation and individualisation of secondary and primary stroke prevention are being discussed.

\section{WAYS TO REDUCE DOSES AND RELATIVE RADIATION RISK, TO PATIENTS, IN INTERVENTIONAL CARDIOLOGY}

\section{Benini A, Pedersen F, Jorgensen E}

Cardiac Catheterization Laboratory, Department of Cardiology, Heart Center, University Hospital Rigshospitalet, Copenhagen, Denmark

There is growing concern about doses delivered to patients in interventional cardiology due to the deterministic and stochastic risks related to X-Rays. The individual doses might be sometimes rather high, certainly above the threshold of deterministic risk. At the same time as the number of patients undertaking this kind of investigation is getting larger and larger there might some concern, also from the point of view of stochastic risk.

The recommendations of the International Committee for Radiation Protection (ICRP) and international Atomic Energy Agency (IAEA) are followed for the evaluation of doses.

The Catheterization Laboratory (Cath Lab), is equipped with seven angiography units, of most recent Philips and Siemens models, the quality of equipment and its working condition are up to high required standards. There is growing concern about doses delivered to patients in interventional cardiology and more effort has been put in limiting the probability of radiation injuries and stochastic effects, with particular attention paid to children. In particular, repeated procedures on the same patient deliver doses in the range of deterministic effect. A detailed analysis of situation is important in order to increase awareness and discuss ways for optimization of the procedures and, when possible, limiting the fluoroscopy time (FT). Each angiography unit has a dose area product (DAP) meter. A computerized clinical database of procedures is active as from 1998, including DAP values and FT. The Cath LAB is well equipped with personal dosimeters, lead aprons, lead eyewear and lead glass shields, for the workers. Data from more than 50.000 diagnostic coronary angiography procedures, more than 20.000 percutaneous coronary intervention (PCI) procedures, and circa 800 congenital heart disease procedures are available in the clinical database.

Data related to the diagnostic procedures and the PCIs are analyzed separately, taking into account: patients' average age and gender, BMI, FT, DAP value, operator (level of experience) and the number of lesions in case of PCI. Doses to patients during PCI were statistically significantly related to body mass index (BMI) of patients and number of PCI lesions (PCI-complexity), as expected, but also to the different operators, and their experience levels.

In addition, circa 800 congenital heart disease procedures (circa 200 patients with more than one intervention) have been analyzed, dividing the patients into four age groups (1-364 days, 1-5 years, 6-11 years and $12-$ 18 years). The exercise to analyze all the above data has been done at the end of 2008 and repeated in March 2011. In conclusions doses to patients during cardiac interventional procedures seem to be within the "expected" dose range. However, a few percent of patients receive relatively high doses. Patients' BMI, procedures' complexity and operators' way-of-work are important factors; this last one, in particular, shows that there is room for improvement in the optimization of procedures and reduction of doses to patients (doses should always be compared to clinical results).

In conclusion, taking into account all the above factors, it is important to put in evidence that, from the comparison of 2008 and 2011 data, it appears clearly that a remarkable improvement has occurred in saving doses to patients during the years 2009 and 2010, due to new technology and awareness on radiation protection. Doses to staff are monitored regularly without any major problem, due to the appropriate use of protection devices.

LARGE VESSEL ANEURISMS IN THE ERA OF GENOMICS AND PROTEOMICS: HORIZONS OF PREDICTIVE MEDICINE

Cherepakhin DI $I^{4}$ Belov $Y V^{1}$, Charchyan $E^{1}$, Suchkov $S V^{2,3}$ 
${ }^{1}$ B.V. Petrovsky Russian Research Center for Surgery (RRCS), Russian Academy of Medical Sciences, Moscow, Russia

${ }^{2}$ I.M. Sechenov First Moscow State Medical University,

Moscow, Russia

${ }^{3}$ Moscow State Medical Dentistry University (MSMDU), Moscow, Russia

${ }^{4}$ N.I. Pirogov Russian State Medical University (RSMU), Moscow, Russia

The difficulties in diagnosis and therapeutic management of LVA (Large vessel) and AA (aortic aneurisms) arise from the lack or vagueness of clinical symptomatology. Patients are usually admitted to a hospital in the critical state. Therefore, a search for novel diagnostic tools for detecting minor or slowly progressing aneurisms is a task of paramount importance. The main triggering factors of LVA and AA include: (i) genetic predisposition; (ii) atherosclerosis and arterial hypertension; (iii) male gender; (iv) smoking; (v) carriage of infectious pathogens (e.g., $T$. pallidum, Chl. pneumonia), etc. Early (prenosologic) diagnosis forms the basis for drug therapy.

We propose a designing of novel efficient technologies for early diagnosis of AA on the basis of molecular changes in human blood and tissues in the course of disease development. In the framework of this approach, state-of-art algorithms of preclinical diagnosis of LVA are generated at the interface of genomics, proteomics and metabolomics.

The key biomarkers for designing advanced diagnostic protocols for LVA are as follows:

(i) Osteoprotegerin (OPG), an early diagnostic marker for AA manifesting high specificity for ang-II-dependent forms of AA.

(ii) Proteases (their metabolic disbalance is the main cause of AA).

Metalloproteases (MPP-2, MPP-9) responsible for elastin destruction in aortic wall tissues play a crucial role in the pathogenesis of AA. Any shift in the equilibrium between activation factors and tissue inhibitors of MMP initiates decomposition of collagen and elastin, which, in its turn, provokes AA. Enhanced activity of TGF-B (transforming growth factor beta) and NFkB (nuclear factor kappa beta) also plays a role in MMP activation. Cystatin-C is widely known as a protease inhibitor; its deficiency was used as a valuable diagnostic marker in our studies.

The first step includes screening blood sera for MMP-2, MMP-9, OPG, cystatin-C, TIMP/MPTI-1, TIMP/MPTI-2, IL-6, TNF-a, TGF-B and NFkB. Their comprehensive analysis is aimed at detecting AA-associated pathologies. The second step includes visualization of the aorta by using contrasting MRT (Magnetic resonance imaging) or MSCT
(Multidetector spiral computed tomography) with the ultimate goal to detect and monitor the dynamics of even minor changes in the aorta diameter.

The third, and technologically most sophisticated, step consists in obtaining diagnostic aortic wall bioptats for immunomorphological analysis with subsequent verification and confirmation of the final diagnosis.

In the state of the art of medical practice, the traditional "attending physician-patient" approach should give way to a more "personalized" ("consulting physician-healthy individual") strategy. Our recent findings provide a way for designing primary preclinical diagnostic protocols for more adequate assessment of aortic wall status and early implementation of preventive drug therapy.

\section{PREDICTIVE TESTING OF THROMBOPHILIA- RELATED GENETIC VARIATIONS IN THE CZECH POPULATION}

Koudová M, Vlčková R, Bittóová M, Majer M, Slepičková I, Lavická E, Hlaváčová M, Izrael G, Indráková V, Karas $J$ GHC Genetics, s.r.o. - Private Medical Facility, Prague, Czech Republic

Introduction: Thrombophilia is a condition generally increasing a predisposition to the formation of clots. It is a multi-factorial disease resulting from a combined effect of genetic predisposition and other factors such as prolonged periods of rest after surgery, trauma to the leg, heart disease, certain cancers and pregnancy, dehydration, smoking, overweight and hormonal contraception. Genetic predisposition of thrombosis includes mutations and genetic variations in genes that disrupt the balance of creation and dissolution of clots and increase a level of homocysteine in the blood.

Methods: 800 males and 800 females from the Czech population were tested in the pilot projects of preventive care supported by VZP ČR (General Health Insurance Company, Czech Republic). The age average of tested patients was 39.9 years. The material of DNA isolation was obtained oral mucosa by flocked Swabs or by taking incoagulable peripheral venous blood. DNA was isolated by using QIAmp DNA Minikit / DNA QuickGene 810 and analyzed by using following methods for molecular genetic analysis: ARMS-PCR, real-time PCR, fragment analysis on ABI 3130 genetic analyzer, High-Resolution Melting analysis and a DNA microarray (GoldenGate genotyping Assay). The following mutations / genetic variants were tested: Leiden (G1691A) gene F5, G20210A gene $F 2$, C677T and A1298C of gene MTHFR, 4G/5G gene PAI-1, c.196C > T gene ITGB3 (HPA1), C10034T gene $F G G$, g. 1253198C $>$ A and g. $1249535 \mathrm{C}>\mathrm{T}$ gene $F 7$ and I / D (287bp) gene $A C E$. Frequencies of alleles and genotypes were statistically evaluated and compared with the data of 
European population. At the same time the results were correlated with patient data and family history.

Results: The frequency of alleles in genes regulating haemostasis correlated well with the data of the European population. Major thrombophilic mutations (Leiden mutation F5 and G20210A F2) were detected in a total of $10.12 \%$ for females and $11.3 \%$ for males. $80 \%$ of these genetically high-risk individuals did not meet the recommended criteria for clinical genetic testing. For nearly half of them external risk factors for thrombosis (obesity, smoking, women using hormonal contraception) were identified and found at significant risk for the development of TEN and cardiovascular disease. In MTHFR gene influencing the level of homocysteine, a triple combination of high risk allele $677 \mathrm{~T}$ a $1298 \mathrm{C}$ (genotypes TT/AC and CT/CC) proved in $0.13 \%$ was found; however, the high risk genotype TT/CC was not identified.

Conclusion: Up to $50 \%$ female / male at significantly increased risk for thrombosis underwent predictive genetic testing. Due to haematological follow up and compliance with recommended preventive measures, we expect a significant reduction in the risk of thrombosis and its complications for the consulted people in the future.

Support: Supported by pilot project of preventive care by VZP Czech Republic known as "Cesta pro zdraví žen" (Way of women's health) and "Cesta ke zdraví mužǔ" (Way of men's health).

\section{AHSG GENE POLYMORPHISM AFFECTS PLASMA LEVELS OF NON-HIGH-DENSITY LIPOPROTEIN CHOLESTEROL IN CAUCASIANS \\ Suchanek $P^{1,2}$, Hubacek $J A^{1,2}$, Kralova Lesna $I^{1,2}$ \\ ${ }^{1}$ Institute for Clinical and Experimental Medicine, Prague, Czech Republic \\ ${ }^{2}$ Cardiovascular Research Centre, Prague, Czech Republic}

Introduction: To evaluate whether the relationship between dietary composition and plasma lipid levels is genetically determined. The alpha2 Heremans-Schmid glycoprotein (AHSG) gene is implicated in the regulation of body fat and insulin sensitivity. The genotype Met/Met (SNP rs4917) was associated with a marked increase in the lipolytic sensitivity to beta2-adrenoreceptor adipocyte on contrary to genotypes $\mathrm{Thr} / \mathrm{Thr}$ and $\mathrm{Thr} / \mathrm{Met}$. We have evaluated the influence of common AHSG gene polymorphism (Thr248Met) on plasma lipid concentrations in $1 \%$ representative sample of the Czech population aged 2565 years

Aim: The influence of polymorphisms of variants Met/Met in the AHSG gene on plasma cholesterol fractions was evaluated in 1152 men and 1345 women representatively selected from the Czech population. Total, low-density lipoprotein cholesterol and HDL cholesterol levels were analyzed.

Results: The Thr/Met variation in the AHSG gene was found to affect plasma on total and LDL cholesterol, showing significantly higher levels in Thr/Thr carriers than in Met/Met homozygotes. This association was observed in both men (total cholesterol $5.76+/-1.03 \mathrm{mmol} / \mathrm{L}$ in $\mathrm{Thr} / \mathrm{Thr}$ carriers versus $5.48+/-0.90 \mathrm{mmol} / \mathrm{L}$ in Met/Met homozygotes; $\mathrm{P}<0.001$ ) and women (total cholesterol $5.77+/-1.17$ $\mathrm{mmol} / \mathrm{L}$ in in $\mathrm{Thr} / \mathrm{Thr}$ carriers versus $5.56+/-0.97 \mathrm{mmol} / \mathrm{L}$ in Met/Met homozygotes; $\mathrm{P}<0.001$ ), similary men LDL cholesterol $3.67+/-0.88 \mathrm{mmol} / \mathrm{L}$ in in $\mathrm{Thr} / \mathrm{Thr}$ carriers versus $3.40+/-0.77 \mathrm{mmol} / \mathrm{L}$ in Met/Met homozygotes; $\mathrm{P}<$ 0.01 ) and women (LDL cholesterol $3.63+/-1.05 \mathrm{mmol} / \mathrm{L}$ in in $\mathrm{Thr} / \mathrm{Thr}$ carriers versus $3.46+/-0.91 \mathrm{mmol} / \mathrm{L}$ in $\mathrm{Met} / \mathrm{Met}$ homozygotes; $\mathrm{P}<0.01$ ).

Conclusion: AHSG gene polymorphism plays a role in the genetic determination of total cholesterol and LDL cholesterol levels.

Support: This project was supported by grant NS 10513-3/ 2009 from the Internal Grant Agency of the Ministry of Health of the Czech Republic.

THE EFFECT OF PERINDOPRIL ON ENDOTHELIN AND NITRICOXIDE PRODUCTION AND CIRCADIAN ALTERATION OF CARDIOVASCULAR INDICIS IN HYPERTENSIVE RATS

Kezeli $T^{l}$, Gongadze $N^{2}$, Sukoian $G^{l}$, Dolidze $N^{l}$, Rukhadze $T^{l}$

${ }^{1}$ Department of Pharmacology, Faculty of Medicine, State University, Tbilisi, Georgia

${ }^{2}$ Department of Pharmacology, State Medical University, Tbilisi, Georgia

Increasing body of evidence suggests an important role of vasoactive peptides and nitric oxide (NO) in homeostatic mechanisms of circulation. The purpose of this study was to estimate the possible involving of endothelin- $1\left(\mathrm{E}_{1}\right)$ and $\mathrm{NO}$ in the modulatory action of perindopril on circadian rhythm of cardiovascular parameters and baroreflex sensitivity (BRS) during development of arterial hypertension (AH). All experiments were carried out in male non-anesthetized freely moving conscious rats with renovascular hypertension (two-kidney, one clip) and in normotensive Wistar Kyoto (WKY) rats weighing 200-250 g. Hypertensive rats (HR) were divided into three groups: 3, 6 and 8 weeks after creation of experimental model of AH. BRS was evaluated by measuring heart period (HP) in response to rises in arterial pressure (AP) $30-50 \mathrm{~mm} \mathrm{Hg}$ above control after i.v. injection of phenylephrine $(5-10 \mathrm{mcg} / \mathrm{kg})$. The slope of relationship between AP and HP was used as an index of BRS. It has been found that during the development of $\mathrm{AH}$ the acrophase of AP compared with normotensive rats 
changed from morning hours $(9.00-12.00 \mathrm{am})$ to evening $(7.00-9.00 \mathrm{pm})$ and night $(10.00-12.00 \mathrm{pm})$ hours. BRS significantly reduced being in 3, 6 and 8 weeks, respectively, HR $0.42 \pm 0.06 ; 0.35 \pm 0.08$ and $0.28 \pm 0.05 \mathrm{~ms} / \mathrm{mm} \mathrm{Hg}$ VS WKY rats $(0.98 \pm 0.1 \overline{6} \mathrm{~ms} / \mathrm{mm} \mathrm{Hg})$ with related changes of its acrophase in contrast to WKY rats from evening and night hours $(7.00-12.00 \mathrm{pm})$ to morning and day hours $(10.00 \mathrm{am}-1.00 \mathrm{pm})$. This alteration of BRS in HR unlike WKY rats has correlated with significant increase of $E_{1}$ plasma concentration especially in 6 and 8 weeks HR, respectively, $6: 1+0,5$ and $7: 8+0,4 \mathrm{pg} / \mathrm{ml}$ VS WKY rats $(2: 6 \pm 0: 2 \mathrm{pg} / \mathrm{ml})$. The increased plasma level of $E_{1}$ in HR simultaneously was accompanied by marked decrease of activity of soluble guanylate cyclase. Estimation of blood pressure and BRS in HR after one-week perindopril administration $(5 \mathrm{mg} / \mathrm{kg}$ i.p. daily) indicated a significant hypotensive effect with increase of BRS, especially in 6 and 8 weeks HR. The pronounced ability of perindopril to correct the disturbances in the circadian variation of AP and BRS has been shown, which correlated in HR with significant decrease of $E_{1}$ plasma level and marked increase of guanylate cyclase activity. The present data suggest the possible impact of $\mathrm{E}_{1}$ and $\mathrm{NO}$ in modulatory effect of perindopril on cardiovascular circadian rhythm and BRS in HR. 